\title{
INFORMATION THEORETIC APPROACH FOR WAVEFORM DESIGN IN COEXISTING MIMO RADAR AND MIMO COMMUNICATIONS
}

\author{
Mohammad Alaee-Kerharoodi, Bhavani Shankar M. R., Kumar Vijay Mishra and Björn Ottersten \\ SnT - Interdisciplinary Centre for Security, Reliability and Trust, University of Luxembourg
}

\begin{abstract}
We investigate waveform design for coexistence between a multipleinput multiple-output (MIMO) radar and MIMO communications (MRMC), with a radar-centric criterion that leads to a minimal interference in the communications system. The communications use the traditional mode of operation in Long Term Evolution (LTE)/Advanced (FDD), where we formulate the design problem based on information-theoretic criterion with the discrete phase constraint at the design stage. The optimization problem, is nonconvex, multi-objective and multi-variable, where we propose an efficient algorithm based on the coordinate descent (CD) framework to simultaneously improve radar target detection performance and the communications rate. The numerical results indicate the effectiveness of the proposed algorithm in designing discrete phase set of sequences, potentially binary.
\end{abstract}

Index Terms - Colocated array, MIMO communications, MIMO radar, MRMC, spectral coexistence

\section{INTRODUCTION}

Extreme crowding of electromagnetic spectrum in recent years has led to emergence of complex challenges in designing radar and communications systems $[1,2]$. Both systems need wide bandwidth to provide a designated quality-of-service (QoS) thus resulting in competing interests in exploiting the spectrum. Current approaches for mitigating this problem are broadly grouped in two classes. In spectral co-design [2,3], a common waveform is employed by the radar and communications system while sharing the hardware resources at the transmit $(\mathrm{Tx})$ and/or receive $(\mathrm{Rx})$. In spectral coexistence, radar and communications individually address the suppression of interference from the other system. The overall architecture usually promotes the performance of only one system leading to a radarcentric $[4,5]$ or communications-centric [6] performance.

There is a vast body of recent literature on spectrum sharing between single antenna communications and monostatic radars. However, coexistence of Multiple Input Multiple Output (MIMO) radar and MIMO communications (MRMC) is a relatively recent development, driven particularly by recent growth in millimeter wave (mmWave) communications and automotive applications [1]. Early works on MRMC proposed null space projection (NSP) beamforming which projects the colocated MIMO radar signals onto the nullspace of the interference channel matrix from radar Tx to MIMO communications $\mathrm{Rx}$; processing techniques included single Base Station (BS) interference mitigation [7], matrix completion [8,9], and switched small singular value space projection (SSVSP) [10]. There are also recent attempts to generalize MRMC systems from

This work was supported by FNR (Luxembourg) through the BRIDGES project "AWARDS", C-PPP17/IS/11827256/AWARDS, and CORE project "SPRINGER", C18/IS/12734677/SPRINGER.

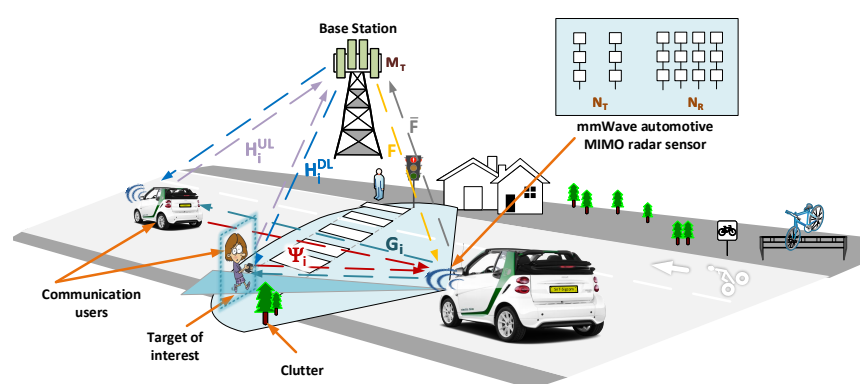

Fig. 1. An illustration of coexistence between MIMO radar and MIMO communications (MRMC). The pedestrian is the target-ofinterest for the MIMO radar deployed on a vehicle on the right. The buildings and vegetation comprise the clutter. At the same time, the pedestrian and different vehicles are equipped with MIMO communications devices that correspond with the nearest BS.

single-user (SU) MIMO communications to multiple-user [11]. In some studies [12,13], only one of the systems - radar or communications - is in the MIMO configuration. These systems are, therefore, not strictly MRMC designs. In [4, 5, 14, 15], optimal space-time transmit waveforms for a colocated MIMO radar that is jointly designed with point-to-point (P2P) MIMO communications code-book is suggested. However, these solutions treat either radar or communications as a primary system and do not yield an equally efficient performance of the secondary system. Contrary to these works, in this paper, we focus on the waveform design for a radarcentric MRMC coexistence that leads to a minimal interference in the communications system.

The Tx amplifiers of the typical radars are naturally powerconstrained implying that the only degree of freedom is the waveform phase. Such a constraint on the waveform optimization makes the problem non-convex resulting in a significantly more complex problem than its unconstrained version [16-20]. Further, the constant modulus waveform design assumes the possibility of defining phase to arbitrary precision. However, in practice, only a finite number of phases are implemented in hardware [16,21], and any quantization to discrete phases would distort the spectral shape. Our solution, on the other hand, is to directly design the discrete phase sequences, by putting the constraint on the design stage.

Throughout this paper, we denote boldface lowercase, boldface uppercase and calligraphic letters for vectors, matrices and index sets, respectively. We denote the transpose and Hermitian by $(\cdot)^{T}$ and $(\cdot)^{H}$, respectively. The Kronecker product is written as $\otimes$. We use $\mathbf{I}_{N}$ for the identity matrix of size $N \times N$. 


\section{SYSTEM ARCHITECTURE AND MODEL}

We consider a colocated MIMO radar comprising $N_{T}$ Tx antennas and $N_{R} \mathrm{Rx}$ antennas, which coexists with MIMO communications with $M_{T}$ Tx and $M_{R} \mathrm{Rx}$ antennas (see Figure 1). Both radar and communications are narrow band, but the radar bandwidth occupies the entire communications bandwidth, including Down Link (DL) and Up Link (UL). The MIMO radar transmits $\overline{\boldsymbol{x}}_{m}=\left[\bar{x}_{m}(1), \bar{x}_{m}(2), \ldots, \bar{x}_{m}(L)\right]^{T} \in \mathbb{C}^{L}$, at $m$-th Tx antenna, where $L$ is the sequence length (intra-pulse coding in Code Division Multiplexing (CDM)-MIMO radar [18]). At $l$-th epoch, $l=1, \ldots, L$, the transmitted waveform by the MIMO radar from all the $N_{T}$ transmit antennas is,

$$
\widetilde{\boldsymbol{x}}_{l}=\left[\bar{x}_{1}(l), \bar{x}_{2}(l), \ldots, \bar{x}_{N_{T}}(l)\right]^{T} \in \mathbb{C}^{N_{T}} .
$$

We suppose that a farfield moving target is located at the direction $\theta_{0}$ with the reflection coefficient $\alpha_{0}$ and normalized relative Doppler frequency $f_{d_{0}}$. Meanwhile, $Q$ clutter patches (signal-dependent interference) are located at $\theta_{1}, \ldots, \theta_{Q}$ with $\alpha_{q}$ reflection coefficients.

The MIMO BS also simultaneously serves multiple communication users by transmitting the symbols

$$
\widetilde{\boldsymbol{s}}_{l}^{\mathcal{B S}}=\left[\widetilde{s}_{1}^{\mathcal{B S}}(l), \widetilde{s}_{2}^{\mathcal{B S}}(l), \ldots, \widetilde{s}_{M_{T}}^{\mathcal{B S}}(l)\right]^{T} \in \mathbb{C}^{M_{T}} .
$$

Without loss of generality, we assume that both communications and radar systems have Uniform Linear Array (ULA) Tx/Rx antennas ${ }^{1}$, and are synchronized in terms of sampling times, when all channels remain fixed over $L$ symbol intervals. We adopt $\boldsymbol{a}_{x}(\alpha)$ to show the steering vectors of the radar system, where we replace $x$ with $T$ or $R$ to indicate Tx or Rx, respectively. We adopt $\widetilde{\boldsymbol{a}}_{x}(\alpha)$ to denote the steering vectors of the communications with superscripts $\mathcal{B S} \mathcal{U}_{i}$ to denote the BS or $i$-th user, respectively.

Assume that $\boldsymbol{F} \in \mathbb{C}^{N_{R} \times M_{T}}$ indicates the channel between BS and radar (see Figure 1), where $\Pi$ paths are available with the departure angles $\phi_{p}, p=1, \ldots, \Pi$ and incident angles $\theta_{p}$, i. e.,

$$
\boldsymbol{F}=\sum_{p=1}^{\Pi} \tau_{p} \boldsymbol{B}\left(\theta_{p}, \phi_{p}\right),
$$

where $\boldsymbol{B}\left(\theta_{p}, \phi_{p}\right)=\boldsymbol{a}_{R}\left(\theta_{p}\right)\left(\widetilde{\boldsymbol{a}}_{T}^{\mathcal{B S}}\left(\phi_{p}\right)\right)^{H}$, and $\tau_{p}$ denotes the unit-mean-square-value fading coefficient of the $p$-th propagation path from BS towards radar. Using the traditional mode of operation in LTE/Advanced (FDD), $i$-th communications user with $\widetilde{M}_{T}^{i}$ Tx antennas also transmits

$$
\widetilde{\boldsymbol{s}}_{l}^{\mathcal{U}_{i}}=\left[\widetilde{s}_{1}^{\mathcal{U}_{i}}(l), \widetilde{s}_{2}^{\mathcal{U}_{i}}(l), \ldots, \widetilde{s}_{\widetilde{M}_{T}^{i}}^{\mathcal{U}_{i}}(l)\right]^{T} \in \mathbb{C}^{\widetilde{M}_{T}^{i}},
$$

where $i=1,2, \ldots, I$, and $I$ stands for number of users. We denote by $\boldsymbol{\Psi}_{i}$ the channel between $i$-th communications user and radar which is defined by, $\boldsymbol{\Psi}_{i}=\sum_{\widetilde{p}=1}^{\widetilde{\Pi}} \kappa_{\widetilde{p}} \widetilde{\boldsymbol{B}}\left(\theta_{\widetilde{p}}, \widetilde{\phi}_{\widetilde{p}}\right)$, where $\kappa_{\widetilde{p}}$ denotes the unit-mean-square-value fading coefficient of the $\widetilde{p}$-th propagation path from $i$-th user towards radar, and

$$
\widetilde{\boldsymbol{B}}\left(\theta_{\widetilde{p}}, \widetilde{\phi}_{\widetilde{p}}\right)=\boldsymbol{a}_{R}\left(\theta_{\widetilde{p}}\right)\left(\widetilde{\boldsymbol{a}}_{T}^{\mathcal{U}_{i}}\left(\widetilde{\phi}_{\widetilde{p}}\right)\right)^{H} \text {, with angle of arrival } \theta_{\widetilde{p}} \text { and }
$$
angle of departure $\widetilde{\phi}_{\widetilde{p}}$.

\subsection{Received signal at radar}

In the MIMO radar receive side, the received complex baseband signal from all $N_{R} \operatorname{Rx}$ antennas at time index $l \in \mathbb{N}_{+}$is given by

\footnotetext{
${ }^{1}$ Assuming ULA, the steering vector at angle $\alpha$ can be obtained by, $\boldsymbol{a}_{x}(\alpha)=\left[1, \ldots, \exp \left(j \frac{2 \pi}{\lambda}\left(N_{x}-1\right) d_{x} \sin (\alpha)\right)\right]^{T}$, where $d_{x}$ indicates the distance between the array elements.
}

$$
\begin{aligned}
\widetilde{\boldsymbol{y}}_{l}^{\mathcal{R}}= & \alpha_{0} e^{j 2 \pi f_{d_{0}}(l-1)} \boldsymbol{A}^{H}\left(\theta_{0}\right) \widetilde{\boldsymbol{x}}_{l}+\sum_{q=1}^{Q} \alpha_{q} \boldsymbol{A}^{H}\left(\theta_{q}\right) \widetilde{\boldsymbol{x}}_{l} \\
& +\boldsymbol{F} \widetilde{\boldsymbol{s}}_{l}^{\mathcal{B S}}+\sum_{i=1}^{I} \boldsymbol{\Psi}_{i} \widetilde{\boldsymbol{s}}_{l}^{\mathcal{U}_{i}}+\widetilde{\boldsymbol{w}}_{l}^{\mathcal{R}}
\end{aligned}
$$

where $\widetilde{\boldsymbol{y}}_{l}^{\mathcal{R}} \in \mathbb{C}^{N_{R}}, \boldsymbol{A}(\theta)=\boldsymbol{a}_{T}(\theta) \boldsymbol{a}_{R}^{H}(\theta) \in \mathbb{C}^{N_{T} \times N_{R}}$, and $\widetilde{\boldsymbol{w}}_{l}^{\mathcal{R}} \in$ $\mathbb{C}^{N_{R}}$ denotes additive noise, modeled as independent and identically distributed (i.i.d.) complex circular zero-mean Gaussian random vector, i.e., $\widetilde{\boldsymbol{w}}_{l}^{\mathcal{R}} \sim \mathcal{C} \mathcal{N}\left(0, \sigma_{\tilde{w} \mathcal{R}}^{2} \boldsymbol{I}_{N_{R}}\right)$.

Rearranging $\boldsymbol{Y}^{\mathcal{R}}=\left[\widetilde{\boldsymbol{y}}_{1}^{\mathcal{R}}, \widetilde{\boldsymbol{y}}_{2}^{\mathcal{R}}, \ldots, \widetilde{\boldsymbol{y}}_{L}^{\mathcal{R}}\right]^{T} \in \mathbb{C}^{L \times N_{R}}$, the received signal from all the $N_{R}$ antennas will be obtained by,

$$
\begin{aligned}
\boldsymbol{Y}^{\mathcal{R}}= & \alpha_{0}\left(\boldsymbol{X} \odot \boldsymbol{P}\left(f_{d_{0}}\right)\right) \boldsymbol{A}\left(\theta_{0}\right)+\boldsymbol{X} \sum_{q=1}^{Q} \alpha_{q} \boldsymbol{A}\left(\theta_{q}\right) \\
& +\boldsymbol{S}^{\mathcal{B S}} \boldsymbol{F}^{T}+\sum_{i=1}^{I} \boldsymbol{S}^{\mathcal{U}_{i}} \boldsymbol{\Psi}_{i}^{T}+\boldsymbol{W}^{\mathcal{R}},
\end{aligned}
$$

where $\boldsymbol{X}=\left[\widetilde{\boldsymbol{x}}_{1}^{\mathcal{R}}, \widetilde{\boldsymbol{x}}_{2}^{\mathcal{R}}, \ldots, \widetilde{\boldsymbol{x}}_{L}^{\mathcal{R}}\right]^{T} \in \mathbb{C}^{L \times N_{T}}$ is the code matrix (set of sequences) that we aim to design in this paper, and $\boldsymbol{P}\left(f_{d}\right)=$ $\left[\boldsymbol{p}_{d}, \ldots, \boldsymbol{p}_{d}\right] \in \mathbb{C}^{L \times N_{R}}$ with Doppler steering vector $\boldsymbol{p}_{d}=$ $\left[1, \ldots, \exp \left(j 2 \pi(L-1) f_{d}\right)\right]^{T}$. Also $\boldsymbol{S}^{\mathcal{B S}}=\left[\widetilde{\boldsymbol{s}}_{1}^{\mathcal{B S}}, \ldots, \widetilde{\boldsymbol{s}}_{L}^{\mathcal{B S}}\right]^{T} \in$

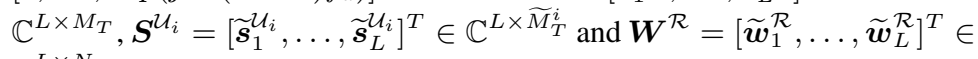
$\mathbb{C}^{L \times N_{R}}$. The covariance matrix of the signal component, namely $\boldsymbol{R}_{x}(\boldsymbol{X})$, can be obtained using the Kronecker properties,

$$
\begin{aligned}
& \mathbb{E}\left\{\operatorname{vec}\left(\alpha_{0}\left(\boldsymbol{X} \odot \boldsymbol{P}\left(f_{d_{0}}\right)\right) \boldsymbol{A}\left(\theta_{0}\right)\right) \operatorname{vec}\left(\alpha_{0}\left(\boldsymbol{X} \odot \boldsymbol{P}\left(f_{d_{0}}\right)\right) \boldsymbol{A}\left(\theta_{0}\right)\right)^{H}\right\} \\
& =\left(\boldsymbol{I}_{N_{R}} \otimes\left[\boldsymbol{X} \odot \boldsymbol{P}\left(f_{d_{0}}\right)\right]\right) \boldsymbol{T}\left(\boldsymbol{I}_{N_{R}} \otimes\left[\boldsymbol{X} \odot \boldsymbol{P}\left(f_{d_{0}}\right)\right]\right)^{H} \in \mathcal{S}_{+}^{L N_{R}},
\end{aligned}
$$

where $\boldsymbol{T}=\sigma_{x}^{2} \operatorname{vec}\left(\boldsymbol{A}\left(\theta_{0}\right)\right) \operatorname{vec}\left(\boldsymbol{A}\left(\theta_{0}\right)\right)^{H} \in \mathbb{C}^{N_{T} N_{R} \times N_{T} N_{R}}$ and $\sigma_{x}^{2}=\mathbb{E}\left\{\alpha_{0} \alpha_{0}^{*}\right\}$.

Similarly, by defining $\boldsymbol{Q}=\sum_{q=1}^{Q} \sigma_{q}^{2} \operatorname{vec}\left(\boldsymbol{A}\left(\theta_{q}\right)\right) \operatorname{vec}\left(\boldsymbol{A}\left(\theta_{q}\right)\right)^{H}$ and $\sigma_{q}^{2}=\mathbb{E}\left\{\alpha_{q} \alpha_{q}^{*}\right\}$, we obtain $\boldsymbol{R}_{c}(\boldsymbol{X})=\left(\boldsymbol{I}_{N_{R}} \otimes \boldsymbol{X}\right) \boldsymbol{Q}\left(\boldsymbol{I}_{N_{R}} \otimes \boldsymbol{X}\right)^{H}$. Further, let $\boldsymbol{Q}^{\mathcal{B S}}=\sum_{p=1}^{\Pi} \sigma_{p}^{2} \operatorname{vec}\left(\boldsymbol{B}\left(\theta_{p}, \phi_{p}\right)\right) \operatorname{vec}\left(\boldsymbol{B}\left(\theta_{p}, \phi_{p}\right)\right)^{H}$ with $\sigma_{p}^{2}=\mathbb{E}\left\{\tau_{p} \tau_{p}^{*}\right\}$, and $\boldsymbol{Q}^{\mathcal{U}_{i}}=\sum_{\widetilde{p}=1}^{\widetilde{\Pi}} \sigma_{\widetilde{p}}^{2} \operatorname{vec}\left(\widetilde{\boldsymbol{B}}\left(\theta_{\widetilde{p}}, \widetilde{\phi}_{\widetilde{p}}\right)\right) \operatorname{vec}\left(\widetilde{\boldsymbol{B}}\left(\theta_{\widetilde{p}}, \widetilde{\phi}_{\widetilde{p}}\right)\right)^{H}$ with $\sigma_{\widetilde{p}}^{2}=\mathbb{E}\left\{\kappa_{\widetilde{p}} \kappa_{\tilde{p}}^{*}\right\}$, the covariance matrix of the interference will be obtained by $\boldsymbol{R}_{\nu}=\left(\boldsymbol{I}_{N_{R}} \otimes \boldsymbol{S}^{\mathcal{B S}}\right) \boldsymbol{Q}^{\mathcal{B S}}\left(\boldsymbol{I}_{N_{R}} \otimes \boldsymbol{S}^{\mathcal{B S}}\right)^{H}$

$+\sum_{i=1}^{I}\left(\boldsymbol{I}_{N_{R}} \otimes \boldsymbol{S}^{\mathcal{U}_{i}}\right) \boldsymbol{Q}^{\mathcal{U}_{i}}\left(\boldsymbol{I}_{N_{R}} \otimes \boldsymbol{S}^{\mathcal{U}_{i}}\right)^{H}$. Further, the covariance matrix of noise is $\boldsymbol{R}_{w}{ }_{\mathcal{R}}=\mathbb{E}\left\{\operatorname{vec}\left(\boldsymbol{W}^{\mathcal{R}}\right) \operatorname{vec}\left(\boldsymbol{W}^{\mathcal{R}}\right)^{H}\right\}$. By creating a vector illustration of the received signal in (5), and defining $\boldsymbol{y}^{\mathcal{R}}=\operatorname{vec}\left(\boldsymbol{Y}^{\mathcal{R}}\right), \boldsymbol{x}=\operatorname{vec}\left(\alpha_{0}\left(\boldsymbol{X} \odot \boldsymbol{P}\left(f_{d_{0}}\right)\right) \boldsymbol{A}\left(\theta_{0}\right)\right)$, $\boldsymbol{c}=\operatorname{vec}\left(\boldsymbol{X} \sum_{q=1}^{Q} \alpha_{q} \boldsymbol{A}\left(\theta_{q}\right)\right), \boldsymbol{\nu}=\operatorname{vec}\left(\boldsymbol{S}^{\mathcal{B S}} \boldsymbol{F}^{T}+\sum_{i=1}^{I} \boldsymbol{S}^{\mathcal{U}_{i}} \boldsymbol{\Psi}_{i}^{T}\right)$, $\boldsymbol{w}^{\mathcal{R}}=\operatorname{vec}\left(\boldsymbol{W}^{\mathcal{R}}\right), \boldsymbol{D}(\boldsymbol{X})=\boldsymbol{R}_{c}(\boldsymbol{X})+\boldsymbol{R}_{\nu}+\boldsymbol{R}_{w \mathcal{R}}, \boldsymbol{\zeta}^{\mathcal{R}}=$ $(\boldsymbol{D}(\boldsymbol{X}))^{-\frac{1}{2}} \boldsymbol{y}^{\mathcal{R}}$, and $\boldsymbol{\Delta}(\boldsymbol{X})=(\boldsymbol{D}(\boldsymbol{X}))^{-\frac{1}{2}} \boldsymbol{R}_{x}(\boldsymbol{X})(\boldsymbol{D}(\boldsymbol{X}))^{-\frac{1}{2}}$, the detection problem with the assumption of Gaussian interference can be written as [20],

$$
\boldsymbol{\zeta}^{\mathcal{R}} \sim \begin{cases}\mathcal{C N}\left(\mathbf{0}, \boldsymbol{I}_{L N_{R}}+\boldsymbol{\Delta}(\boldsymbol{X})\right) & : H_{1} \\ \mathcal{C N}\left(\mathbf{0}, \boldsymbol{I}_{L N_{R}}\right) & : H_{0}\end{cases}
$$

Using the eigen decomposition $\boldsymbol{\Delta}(\boldsymbol{X})=\widetilde{\boldsymbol{U}}^{H} \boldsymbol{\Lambda} \widetilde{\boldsymbol{U}}$ with $\boldsymbol{\Lambda}$ being diagonal matrix containing eigenvalues of $\boldsymbol{\Delta}(\boldsymbol{X})$ called $\lambda_{n}$, the optimal Neyman-Pearson (NP) detector of (7) is given by $\sum_{n=1}^{L N_{R}} \frac{\lambda_{n}\left|z_{n}\right|^{2}}{1+\lambda_{n}} \underset{\mathrm{H}_{0}}{\gtrless} \eta$, where $\boldsymbol{z}=\left[z_{1}, \cdots, z_{L N_{R}}\right]^{T}=\widetilde{\boldsymbol{U}}^{H} \boldsymbol{\zeta}^{\mathcal{R}}$ with columns of $\widetilde{\boldsymbol{U}}$ are the associated eigenvectors. The above detector is optimal in the sense that it maximizes probability of 
detection while keeping probability of false-alarm below a certain threshold. However, in most cases, finding the best set of sequences $\boldsymbol{X}$ that maximizes the performance of the optimal detector is not analytically tractable. In such cases, other design criteria referred to as information-theoretic criteria like mutual information $(\mathcal{M})$ can be employed for transmit sequence design [20]. The $\mathcal{M}$ metric associated with (7) is given by,

$$
\mathcal{M}(\boldsymbol{X})=\log \operatorname{det}\left(\boldsymbol{I}_{L N_{R}}+\boldsymbol{\Delta}(\boldsymbol{X})\right) .
$$

\subsection{Received signal at communications}

The communication symbols transmitted from the BS in the DL will be received at the $i$-th user with angle of arrival $\widetilde{\phi}_{d}$ and angle of departure $\phi_{d}$, after passing through the flat frequency channel $\boldsymbol{H}_{i}^{D L} \in$ $\mathbb{C}^{\widetilde{M}_{R}^{i} \times M_{T}}$ (see Figure 1), defined by $\boldsymbol{H}_{i}^{D L}=\sum_{d=1}^{\Delta_{i}} \delta_{d} \overline{\boldsymbol{J}}_{i}\left(\widetilde{\phi}_{d}, \phi_{d}\right)$, where $\overline{\boldsymbol{J}}_{i}\left(\widetilde{\phi}_{d}, \phi_{d}\right)=\widetilde{\boldsymbol{a}}_{R}^{\mathcal{U}_{i}}\left(\widetilde{\phi}_{d}\right)\left(\widetilde{\boldsymbol{a}}_{T}^{\mathcal{B S}}\left(\phi_{d}\right)\right)^{H}$, and $\delta_{d}$ denotes the unit-mean-square-value fading coefficient of the $d$-th propagation path from BS towards $i$-th user.

Additionally, the transmit waveform of the radar will passes through the channel $\boldsymbol{G}_{i} \in \mathbb{C}^{\widetilde{M}_{R}^{i} \times N_{T}}$ and interferes with the communication symbols at the receiver of $i$-th user. Considering $\Omega_{i}$-path channels between radar and $i$-th user, and defining $\overline{\boldsymbol{G}}_{i}\left(\widetilde{\phi}_{k}, \theta_{k}\right)=$ $\widetilde{\boldsymbol{a}}_{R}^{\mathcal{U}_{i}}\left(\widetilde{\phi}_{k}\right) \boldsymbol{a}_{T}^{H}\left(\theta_{k}\right)$, the channel matrix $\boldsymbol{G}_{i}$ can be written as, $\boldsymbol{G}_{i}=$ $\sum_{k=1}^{\Omega_{i}} \beta_{k} \overline{\boldsymbol{G}}_{i}\left(\widetilde{\phi}_{k}, \theta_{k}\right)$, where $\beta_{k}$ denotes the unit-mean-square-value fading coefficient of the $k$-th propagation path from radar towards the $i$-th communication user, and $\widetilde{\boldsymbol{a}}_{R}^{\mathcal{U}_{i}}\left(\widetilde{\phi}_{k}\right)$ is the receive steering vector of $i$-th communication user with angle of arrival $\widetilde{\phi}_{k}$ and angle of departure $\theta_{k}$ from radar transmit array with steering vector $\boldsymbol{a}_{T}\left(\theta_{k}\right)$. Received signal of the $i$-th communication receiver at time sample $l$ is

$$
\widetilde{\boldsymbol{y}}_{l}^{\mathcal{U}_{i}}=\boldsymbol{H}_{i}^{D L} \widetilde{\boldsymbol{s}}_{l}^{\mathcal{B S}}+\boldsymbol{G}_{i} \widetilde{\boldsymbol{x}}_{l}+\widetilde{\boldsymbol{w}}_{l}^{\mathcal{U}_{i}}
$$

where $\widetilde{\boldsymbol{w}}_{l}^{\mathcal{U}_{i}} \in \mathbb{C}^{\widetilde{M}_{R}^{i}}$ denotes additive noise, modeled as $\widetilde{\boldsymbol{w}}_{l}^{\mathcal{U}_{i}} \sim$ $\mathcal{C N}\left(0, \sigma_{\tilde{w}^{i}}^{2} \boldsymbol{I}_{\widetilde{M}_{R}^{i}}\right)$.

At the BS, the communication symbols will be received from all active users, while passing thought the frequency flat channels $\boldsymbol{H}_{i}^{U L} \in \mathbb{C}^{M_{R} \times \widetilde{M}_{T}^{i}}, i=1, \ldots, I$. Additionally, the transmit waveform of the radar interferes with the received signal at the BS when passes through the channel $\overline{\boldsymbol{F}} \in \mathbb{C}^{M_{R} \times N_{T}}$. The received signal at the BS in time index $l$ can be expressed as,

$$
\widetilde{\boldsymbol{y}}_{l}^{\mathcal{B S}}=\sum_{i=1}^{I} \boldsymbol{H}_{i}^{U L} \widetilde{\boldsymbol{s}}_{l}^{\mathcal{U}_{i}}+\overline{\boldsymbol{F}} \widetilde{\boldsymbol{x}}_{l}+\widetilde{\boldsymbol{w}}_{l}^{\mathcal{B S}}
$$

where $\widetilde{\boldsymbol{w}}_{l}^{\mathcal{B S}} \in \mathbb{C}^{M_{R}}$ denotes additive noise, modeled as $\widetilde{\boldsymbol{w}}_{l}^{\mathcal{B S}} \sim$ $\mathcal{C N}\left(0, \sigma_{\tilde{w}^{\mathcal{B} S}}^{2} \boldsymbol{I}_{M_{R}}\right)$. Also, $\boldsymbol{H}_{i}^{U L}=\sum_{\widetilde{d}_{i}=1}^{\widetilde{\delta}_{\tilde{d}}} \widetilde{\delta}_{\widetilde{\boldsymbol{J}}}\left(\widetilde{\phi}_{\widetilde{d}}, \widetilde{\phi}_{\widetilde{d}}\right)$, where $\widetilde{\boldsymbol{J}}_{i}\left(\phi_{\tilde{d}}, \widetilde{\phi}_{\widetilde{d}}\right)=\widetilde{\boldsymbol{a}}_{R}^{\mathcal{B S}}\left(\phi_{\widetilde{d}}\right)\left(\widetilde{\boldsymbol{a}}_{T}^{\mathcal{U}_{i}}\left(\widetilde{\phi}_{\widetilde{d}}\right)\right)^{H}$, and $\widetilde{\delta}_{\widetilde{d}}$ denotes the unitmean-square-value fading coefficient of the $\widetilde{d}$-th propagation path from $i$-th user towards BS. Similarly, by denoting $\gamma_{\widetilde{k}}$ as the unitmean-square-value fading coefficient of the $\widetilde{k}$-th propagation path from radar towards the BS and considering $\widetilde{\Omega}$ path between radar and BS, the channel matrix $\overline{\boldsymbol{F}} \in \mathbb{C}^{M_{R} \times N_{T}}$ can be written as, $\overline{\boldsymbol{F}}=$ $\sum_{\widetilde{k}=1}^{\widetilde{\Omega}} \gamma_{\widetilde{k}} \widetilde{\boldsymbol{F}}\left(\phi_{\widetilde{k}}, \theta_{\widetilde{k}}\right)$, where $\widetilde{\boldsymbol{F}}\left(\phi_{\widetilde{k}}, \theta_{\widetilde{k}}\right)=\widetilde{\boldsymbol{a}}_{R}^{\mathcal{B S}}\left(\phi_{\widetilde{k}}\right) \boldsymbol{a}_{T}^{H}\left(\theta_{\widetilde{k}}\right)$. Similar to the radar receive side, rearranging $\boldsymbol{Y}^{\mathcal{U}_{i}}=\left[\widetilde{\boldsymbol{y}}_{l}^{\mathcal{U}_{1}}, \widetilde{\boldsymbol{y}}_{l}^{\mathcal{U}_{2}}, \ldots, \widetilde{\boldsymbol{y}}_{L}^{\mathcal{U}_{i}}\right]^{T}$
$\mathbb{C}^{L \times \widetilde{M}_{R}^{i}}$ and $\boldsymbol{Y}^{\mathcal{B S}}=\left[\widetilde{\boldsymbol{y}}_{l}^{\mathcal{B S}}, \widetilde{\boldsymbol{y}}_{l}^{\mathcal{B S}}, \ldots, \widetilde{\boldsymbol{y}}_{L}^{\mathcal{B S}}\right]^{T} \in \mathbb{C}^{L \times M_{R}}$, the received signal from all receive antennas respectively at the communication users and $\mathrm{BS}$ can be written as,

$$
\begin{gathered}
\boldsymbol{Y}^{\mathcal{U}_{i}}=\boldsymbol{S}^{\mathcal{B S}}\left(\boldsymbol{H}_{i}^{D L}\right)^{T}+\boldsymbol{X} \boldsymbol{G}_{i}^{T}+\boldsymbol{W}^{\mathcal{U}_{i}}, \\
\boldsymbol{Y}^{\mathcal{B S}}=\sum_{i=1}^{I} \boldsymbol{S}^{\mathcal{U}_{i}}\left(\boldsymbol{H}_{i}^{U L}\right)^{T}+\boldsymbol{X} \overline{\boldsymbol{F}}^{T}+\boldsymbol{W}^{\mathcal{B S}},
\end{gathered}
$$

where $\boldsymbol{S}^{\mathcal{B S}}, \boldsymbol{S}^{\mathcal{U}_{i}}, \boldsymbol{X}$ are defined previously below (5), and $\boldsymbol{W}^{\mathcal{U}_{i}}=$ $\left[\widetilde{\boldsymbol{w}}_{1}^{\mathcal{U}_{i}}, \ldots, \widetilde{\boldsymbol{w}}_{L}^{\mathcal{U}_{i}}\right]^{T} \in \mathbb{C}^{L \times \widetilde{M}_{R}^{i}}, \boldsymbol{W}^{\mathcal{B S}}=\left[\widetilde{\boldsymbol{w}}_{1}^{\mathcal{B S}}, \ldots, \widetilde{\boldsymbol{w}}_{L}^{\mathcal{B S}}\right]^{T} \in$ $\mathbb{C}^{L \times M_{R}}$. Let us assume that the communication symbols transmitted by every user are independent from the each other. The signal covariance matrices at $i$-th user and BS respectively can be obtained by, $\boldsymbol{R}_{\boldsymbol{S}}^{\mathcal{U}_{i}}=\left(\boldsymbol{I}_{\widetilde{M}_{R}^{i}} \otimes \boldsymbol{S}^{\mathcal{B S}}\right) \widetilde{\boldsymbol{Q}}^{\mathcal{B S}}\left(\boldsymbol{I}_{\widetilde{M}_{R}^{i}} \otimes \boldsymbol{S}^{\mathcal{B S}}\right)^{H}$, $\boldsymbol{R}_{\boldsymbol{S}}^{\mathcal{B S}}=\sum_{i=1}^{I}\left(\boldsymbol{I}_{M_{R}} \otimes \boldsymbol{S}^{\mathcal{U}_{i}}\right) \widetilde{\boldsymbol{Q}}^{\mathcal{U}_{i}}\left(\boldsymbol{I}_{M_{R}} \otimes \boldsymbol{S}^{\mathcal{U}_{i}}\right)^{H}$, with $\widetilde{\boldsymbol{Q}}^{\mathcal{B \mathcal { S }}}=$ $\sum_{d=1}^{\Delta_{i}} \sigma_{d}^{2} \operatorname{vec}\left(\overline{\boldsymbol{J}}_{i}\left(\widetilde{\phi}_{d}, \phi_{d}\right)\right) \operatorname{vec}\left(\overline{\boldsymbol{J}}_{i}\left(\widetilde{\phi}_{d}, \phi_{d}\right)\right)^{H}$ and $\quad \widetilde{\boldsymbol{Q}}^{\mathcal{U}_{i}}=$ $\sum_{\widetilde{d}=1}^{\widetilde{\Delta}_{i}} \sigma_{\widetilde{d}}^{2} \operatorname{vec}\left(\widetilde{\boldsymbol{J}}_{i}\left(\phi_{\widetilde{d}}, \widetilde{\phi}_{\widetilde{d}}\right)\right) \operatorname{vec}\left(\widetilde{\boldsymbol{J}}_{i}\left(\phi_{\tilde{d}}, \widetilde{\phi}_{\widetilde{d}}\right)\right)^{H}$ with $\sigma_{d}^{2}=\mathbb{E}\left\{\delta_{d} \delta_{d}^{*}\right\}$ and $\sigma_{\widetilde{d}}^{2}=\mathbb{E}\left\{\widetilde{\delta}_{\widetilde{d}} \widetilde{\delta}_{\widetilde{d}}^{*}\right\}$.

The radar waveform interferes with the communication symbols at both user and BS receive sides. The covariance of interference plus noise in the receiver of $i$-th user of the communications can be expressed as, $\boldsymbol{R}_{C i n}^{\mathcal{U}_{i}}(\boldsymbol{X})\left(\boldsymbol{I}_{\widetilde{M}_{R}^{i}} \otimes \boldsymbol{X}\right) \widetilde{\boldsymbol{\Upsilon}}_{i}\left(\boldsymbol{I}_{\widetilde{M}_{R}^{i}} \otimes \boldsymbol{X}\right)^{H}+$ $\sigma_{\tilde{w}^{\mathcal{U}^{i}}}^{2} \boldsymbol{I}_{L \widetilde{M}_{R}^{i}}$, with $\widetilde{\boldsymbol{\Upsilon}}_{i}=\sum_{k=1}^{\Omega_{i}} \sigma_{k}^{2} \operatorname{vec}\left(\overline{\boldsymbol{G}}_{i}\left(\widetilde{\phi}_{k}, \theta_{k}\right)\right) \operatorname{vec}\left(\overline{\boldsymbol{G}}_{i}\left(\widetilde{\phi}_{k}, \theta_{k}\right)\right)^{H}$, where $\sigma_{k}^{2}$ denotes the interference power in the receiver of $i$-th communication user. The covariance of interference plus noise in the receiver of BS can be written as, $\boldsymbol{R}_{C i n}^{\mathcal{B S}}(\boldsymbol{X})=\left(\boldsymbol{I}_{M_{R}} \otimes \boldsymbol{X}\right) \widetilde{\boldsymbol{\Xi}}\left(\boldsymbol{I}_{M_{R}} \otimes \boldsymbol{X}\right)^{H}+$ $\sigma_{\tilde{w} \mathcal{B S}}^{2} \boldsymbol{I}_{L M_{R}}$, with $\widetilde{\boldsymbol{\Xi}}=\sum_{\widetilde{k}=1}^{\widetilde{\Omega}} \sigma_{\widetilde{k}}^{2} \operatorname{vec}\left(\widetilde{\boldsymbol{F}}\left(\phi_{\widetilde{k}}, \theta_{\widetilde{k}}\right)\right) \operatorname{vec}\left(\widetilde{\boldsymbol{F}}\left(\phi_{\widetilde{k}}, \theta_{\widetilde{k}}\right)\right)^{H}$, where $\sigma_{\widetilde{k}}^{2}$ denotes the interference power in the BS receiver. Let $\Theta^{\mathcal{U}_{i}}(\boldsymbol{X})=\left(\boldsymbol{R}_{\text {Cin }}^{\mathcal{U}_{i}}(\boldsymbol{X})\right)^{-1} \boldsymbol{R}_{\boldsymbol{S}}^{\mathcal{U}_{i}}, \Theta^{\mathcal{B S}}(\boldsymbol{X})=\left(\boldsymbol{R}_{\text {Cin }}^{\mathcal{B S}}(\boldsymbol{X})\right)^{-1} \boldsymbol{R}_{\boldsymbol{S}}^{\mathcal{B} \mathcal{S}}$, a lower bound for the rate per channel use per Degrees of Freedom (DOF), when the codeword is Gaussian can be respectively achieved by $[4,22]$,

$$
\begin{aligned}
& C^{\mathcal{U}_{i}}(\boldsymbol{X}) \triangleq \frac{1}{L} \log \operatorname{det}\left(\boldsymbol{I}_{L \widetilde{M}_{R}^{i}}+\boldsymbol{\Theta}^{\mathcal{U}_{i}}(\boldsymbol{X})\right) \\
& C^{\mathcal{B S}}(\boldsymbol{X}) \triangleq \frac{1}{L} \log \operatorname{det}\left(\boldsymbol{I}_{L M_{R}}+\boldsymbol{\Theta}_{i}^{\mathcal{B S}}(\boldsymbol{X})\right) .
\end{aligned}
$$

\section{THE PROPOSED METHOD}

We aim to maximize the $\mathcal{M}(\boldsymbol{X}), C^{\mathcal{U}_{i}}(\boldsymbol{X})$ and $C^{\mathcal{B S}}(\boldsymbol{X})$, by designing the radar transmit set of sequences $\boldsymbol{X}$, while every entry is adopted from the discrete phase set $\Gamma_{M}=\left\{0, \frac{2 \pi}{M}, \ldots, \frac{2 \pi(M-1)}{M}\right\}$, with $M$ indicating the alphabet size. Indicating $(l, m)$-th entry of the code matrix $\boldsymbol{X}$ by $X_{l, m}, l=1, \ldots, L, m=1, \ldots, N_{T}$, the optimization problem can be written as,

$$
\mathcal{P}_{\text {MRMC }} \begin{cases}\max _{\boldsymbol{X}} & \left\{\mathcal{M}(\boldsymbol{X}), C^{\mathcal{U}_{i}}(\boldsymbol{X}), C^{\mathcal{B S}}(\boldsymbol{X})\right\} \\ \text { subject to } & \arg \left(X_{l, m}\right) \in \Gamma_{M}\end{cases}
$$

which is multi-objective, multi-variable, constrained and nonconvex. In a multi-objective optimization problem, usually a feasible solution that minimizes all the objective functions simultaneously $\in$ does not exist $[17,18]$. A viable means to handle these type of 
problems, is to use the scalarization technique which exploits as objective a specific weighted sum between the objective functions (Pareto-optimization). Particularly, a scalarization of (15) is,

$$
\widetilde{\mathcal{P}}_{\text {MRMC }} \begin{cases}\max _{\boldsymbol{X}} & w_{1} \mathcal{M}(\boldsymbol{X})+w_{2} C^{\mathcal{U}_{i}}(\boldsymbol{X})+w_{3} C^{\mathcal{B S}}(\boldsymbol{X}) \\ \text { subject to } & \arg \left(X_{l, m}\right) \in \Gamma_{M}\end{cases}
$$

where the weighting coefficients $w_{1}, w_{2}, w_{3} \in[0,1]$ and $\sum_{i=1}^{3} w_{i}=$ 1 . The parameters $w_{i}$ trades-off the radar performance and the communication performance. In fact, the different values of $w_{i}$ can lead to various sets of sequences with different goodness, which is an important property for the coexisting systems. An example is automotive MIMO radar, where the performance can be enhanced through a continuous and coordinated feedback between the transmitter and receiver which implies a dynamic adaptation of the sensor's algorithms to the operational context and environmental replies.

To tackle Problem $\widetilde{\mathcal{P}}_{\text {MRMC }}$, we resort to the Coordinate Descent (CD) framework, which has gained growing popularity in various applications due to its straightforward structure and simplicity [17, 18]. In this sense, in a first step we rewrite the objective function based on one variable, keeping fixed the others. Then, we impose the constraint directly on the selected variable, i. e., converting the constrained optimization problem to unconstrained. Let us denote the objective function in $\widetilde{\mathcal{P}}_{\text {MRMC }}$ by $f(\boldsymbol{X})=w_{1} \mathcal{M}(\boldsymbol{X})+w_{2} C^{\mathcal{U}_{i}}(\boldsymbol{X})+$ $w_{3} C^{\mathcal{B S}}(\boldsymbol{X})$. We pick a coordinate $\tilde{l}, \tilde{m}$ from $\widetilde{l}=1, \ldots, L$ and $\widetilde{m}=1, \ldots, N_{T}$, and design the code entry $X_{\widetilde{l}, \widetilde{m}}$, by keeping fixed the other entries of the code matrix $\boldsymbol{X}$. As to the constraint, we directly examine the objective function by putting $X_{\widetilde{l}, \widetilde{m}}$ equal to all the entries of $\Gamma_{M}$, and select the one who maximizes it, as the optimal $X_{\tilde{l}, \widetilde{m}}^{\star}$. Once all the entries of $\boldsymbol{X}$ were updated, we check the the objective function in comparison with the previous iteration ${ }^{2}$, and stop the algorithm if the change was less than a desired threshold. Otherwise, we put the obtained code matrix as the input to the next iteration. Finally, while the iteration increases, we iteratively obtain different sets of optimal sequences, namely $\boldsymbol{X}^{(1)}, \boldsymbol{X}^{(2)}, \ldots, \boldsymbol{X}^{(\star)}$.

\section{PERFORMANCE ANALYSIS}

We consider a MIMO radar with $N_{T}=3 \mathrm{Tx}, N_{R}=4 \mathrm{Rx}$ antennas, and code length $L=11$. We assume a target is located at the angle $\theta_{0}=25$ degrees with normalized Doppler shift $f_{d_{0}}=0.25$; also, the number of interfering clutter patches is supposed to be $Q=7$ around $\theta_{0}$, viz. $\theta_{q}=\{22,23,24,25,26,27,28\}$. A homogenous clutter environment is considered implying that $\sigma_{1}=\sigma_{2}, \ldots,=$ $\sigma_{Q}$. Also, a zero mean white Gaussian noise with $\sigma_{\boldsymbol{w}^{\mathcal{R}}}^{2}=1$ is considered at the radar receiver. We consider $-10 \mathrm{~dB}$ Signal to Noise Ratio (SNR) for the target of interest, while the Clutter to Noise Ratio (CNR) and Interfernece to Noise Ratio (INR) at the range bin of the target are $20 \mathrm{~dB}, 30 \mathrm{~dB}$, respectively ${ }^{3}$.

We also consider BS with $M_{T}=M_{R}=8 \mathrm{Tx}$ and Rx antennas, while $I=10$ active users are served by the BS. Every user, has a single Tx and Rx antenna. Without loss of generality, we assume 5 paths are available between every two systems, i. e., BS and radar, BS and users, users and radar, and vice versa. Further, we randomly select the incident and departure angles between -90 to 90

\footnotetext{
${ }^{2}$ Iteration increases when all the entries of $\boldsymbol{X}$ were updated.

${ }^{3}$ Assuming $\sigma_{s}^{2}, \sigma_{n}^{2}, \sigma_{c}^{2}$, and $\sigma_{\nu}^{2}$ indicating the power of signal, noise, clutter and interference, then $\mathrm{SNR}=\frac{\sigma_{s}^{2}}{\sigma_{n}^{2}}, \mathrm{CNR}=\frac{\sigma_{c}^{2}}{\sigma_{n}^{2}}$, and INR $=\frac{\sigma_{\nu}^{2}}{\sigma_{n}^{2}}$
}

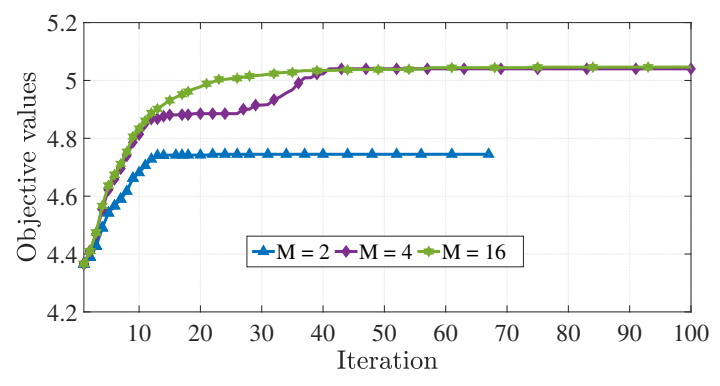

Fig. 2. Convergence behaviour of the proposed method at different alphabet sizes, initializing from uncoded waveform.

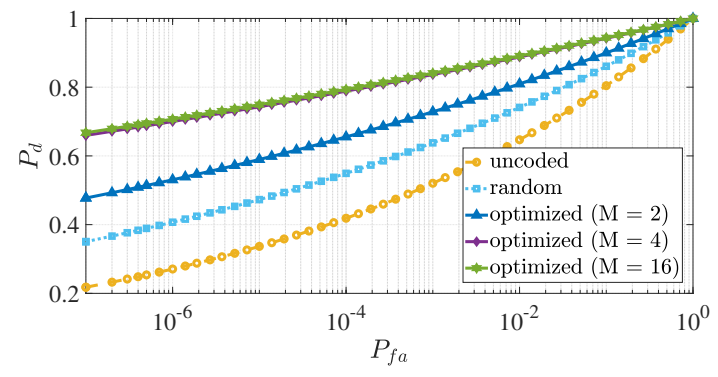

Fig. 3. ROC for uncoded, random and optimized set of sequences at alphabet sizes $M=[2,4,16]$.

degrees. At the communications, we uniformly select SNR of every user in the interval $[5,25] \mathrm{dB}$ and INR in the interval $[10,30] \mathrm{dB}$. We consider similar SNR/INR values for DL and UL paths. In all the simulations, we set $w_{1}=0.5, w_{2}=0.25, w_{3}=0.25$, alphabet sizes $M=\{2,4,16\}$, and the stopping threshold to 0.0001 . We use uncoded $^{4}$ waveforms (which implies phased array transmission) as the initial set of sequences, and the benchmark.

In Figure 2, the convergence curves illustrate the monotonic increasing behavior of the objective function. Interestingly, the objective function is case of $M=4$ is very close to that of obtained with $M=16$. This indicates that with a small values of the alphabet sizes, it would be possible to obtain sequences with promising objective values. It can be further validated in Figure 3, where receiver operating characteristic curve (ROC) curves of the obtained sequences are plotted.

\section{CONCLUSION}

In this paper, we considered MIMO communications with multiple active users working under mode of LTE advanced operation coexisting with MIMO radar and sharing the spectrum. Using the considered scenario, we modeled all the channels between radar, BS, and users, and obtained the different covariance matrices. Also, we evaluated the performances based on mutual information and communication rates. The design problem, was to maximize the rates and the radar performance simultaneously, by designing radar set of sequences. The optimization framework, was non-convex, where we used CD framework to tackle it. A future track is to consider uncertainties on the information, about the channels, clutter, and signal covariance matrices.

${ }^{4} \mathrm{By}$ the uncoded we mean that the phase of the all entries of the set of sequence is the same, preferably 0 . 


\section{REFERENCES}

[1] K. V. Mishra, M. R. Bhavani Shankar, V. Koivunen, B. Ottersten, and S. A. Vorobyov, "Toward millimeter wave joint radar communications: A signal processing perspective," IEEE Signal Processing Magazine, vol. 36, pp. 100-114, 2019.

[2] S. H. Dokhanchi, B. S. Mysore, K. V. Mishra, and B. Ottersten, "A mmWave automotive joint radar-communications system," IEEE Transactions on Aerospace and Electronic Systems, vol. 55, pp. 1241-1260, 2019.

[3] G. Duggal, S. Vishwakarma, K. V. Mishra, and S. S. Ram, "Doppler-resilient 802.11ad-based ultra-short range automotive radar," arXiv preprint arXiv:1902.01306, 2019.

[4] J. Qian, M. Lops, L. Zheng, X. Wang, and Z. He, "Joint system design for coexistence of MIMO radar and MIMO communication," IEEE Transactions on Signal Processing, vol. 66, no. 13, pp. 3504-3519, 2018.

[5] M. Alaee-Kerahroodi, K. V. Mishra, M. R. Bhavani Shankar, and B. Ottersten, "Discrete phase sequence design for coexistence of MIMO radar and MIMO communications," in IEEE International Workshop on Signal Processing Advances in Wireless Communications, 2019, pp. 1-5.

[6] A. Ayyar and K. V. Mishra, "Robust communications-centric coexistence for turbo-coded OFDM with non-traditional radar interference models," in IEEE Radar Conference, 2019, in press.

[7] A. Khawar, A. Abdelhadi, and C. Clancy, "Target detection performance of spectrum sharing MIMO radars," IEEE Sensors Journal, vol. 15, no. 9, pp. 4928-4940, 2015.

[8] B. Li, A. P. Petropulu, and W. Trappe, "Optimum co-design for spectrum sharing between matrix completion based MIMO radars and a MIMO communication system," IEEE Transactions on Signal Processing, vol. 64, no. 17, pp. 4562-4575, 2016.

[9] K. V. Mishra, A. Kruger, and W. F. Krajewski, "Compressed sensing applied to weather radar," in IEEE International Geoscience and Remote Sensing Symposium, 2014, pp. 1832-1835.

[10] J. A. Mahal, A. Khawar, A. Abdelhadi, and T. C. Clancy, "Spectral coexistence of MIMO radar and MIMO cellular system," IEEE Transactions on Aerospace and Electronic Systems, vol. 53, no. 2, pp. 655-668, 2017.

[11] F. Liu, C. Masouros, A. Li, H. Sun, and L. Hanzo, "MUMIMO communications with MIMO radar: From co-existence to joint transmission," IEEE Transactions on Wireless Communications, vol. 17, no. 4, pp. 2755-2770, 2018.

[12] K. Singh, S. Biswas, T. Ratnarajah, and F. A. Khan, "Transceiver design and power allocation for full-duplex MIMO communication systems with spectrum sharing radar," IEEE Transactions on Cognitive Communications and Networking, vol. 4, no. 3, pp. 556-566, 2018.

[13] E. Grossi, M. Lops, and L. Venturino, "Joint design of surveillance radar and MIMO communication in cluttered environments," arXiv preprint arXiv:1907.07943, 2019.

[14] F. Liu, L. Zhou, C. Masouros, A. Li, W. Luo, and A. Petropulu, "Toward dual-functional radar-communication systems: Optimal waveform design," IEEE Transactions on Signal Processing, vol. 66, no. 16, pp. 4264-4279, 2018.
[15] M. Rihan and L. Huang, "Optimum co-design of spectrum sharing between MIMO radar and MIMO communication systems: An interference alignment approach," IEEE Transactions on Vehicular Technology, vol. 67, no. 12, pp. $11667-$ $11680,2018$.

[16] W. Rowe, P. Stoica, and J. Li, "Spectrally constrained waveform design [SP Tips Tricks]," IEEE Signal Processing Magazine, vol. 31, no. 3, pp. 157-162, 2014.

[17] M. Alaee-Kerahroodi, A. Aubry, A. De Maio, M. M. Naghsh, and M. Modarres-Hashemi, "A coordinate-descent framework to design low PSL/ISL sequences," IEEE Transactions on Signal Processing, vol. 65, no. 22, pp. 5942-5956, 2017.

[18] M. Alaee-Kerahroodi, M. Modarres-Hashemi, and M. M. Naghsh, "Designing sets of binary sequences for MIMO radar systems," IEEE Transactions on Signal Processing, vol. 67, no. 13, pp. 3347-3360, 2019.

[19] J. Li and P. Stoica, "MIMO radar with colocated antennas," IEEE Signal Processing Magazine, vol. 24, no. 5, pp. 106-114, 2007.

[20] M. M. Naghsh, M. Modarres-Hashemi, M. Alaee-Kerahroodi, and E. H. M. Alian, "An information theoretic approach to robust constrained code design for MIMO radars," IEEE Transactions on Signal Processing, vol. 65, no. 14, pp. 3647-3661, 2017.

[21] W. Melvin and J. Scheer, Principles of modern radar: Advanced techniques. IET, 2012.

[22] J. Qian, F. Tian, N. Huang, and T. Liu, "Multiobjective optimization for spectral coexistence of radar and communication system," in IEEE Radar Conference, 2019, pp. 1-5. 